\title{
Spectral properties of the planar $t-J$ model
}

\author{
J. Jaklič ${ }^{1}$ and P. Prelovšek ${ }^{1,2}$ \\ ${ }^{1}$ J. Stefan Institute, University of Ljubljana, 1001 Ljubljana, Slovenia \\ ${ }^{2}$ Institut Romand de Recherche Numérique en Physique des Matériaux (IRRMA), PHB-Ecublens, CH-1015 Lausanne, \\ Switzerland
}

(March 25, 2018)

\begin{abstract}
The single-particle spectral functions $A(\mathbf{k}, \omega)$ and self-energies $\Sigma(\mathbf{k}, \omega)$ are calculated within the $t-J$ model using the finite-temperature Lanczos method for small systems. A remarkable asymmetry between the electron and hole part is found. The hole (photoemission) spectra are overdamped, with $\operatorname{Im} \Sigma \propto \omega$ in a wide energy range, consistent with the marginal Fermi liquid scenario, and in good agreement with experiments on cuprates. In contrast, the quasiparticles in the electron part of the spectrum show weak damping.
\end{abstract}

PACS numbers: 71.27.+a, 79.60.-i, 71.20.-b

The normal state of superconducting cuprates in many aspects contradicts the phenomenology of the normal Fermi liquid (FL). Anomalous frequency and temperature dependence of several response functions is generally attributed to electronic correlations, yet a proper description is missing so far. The angle resolved photoemission (ARPES) experiments [1] probe the one-particle spectral function $A(\mathbf{k}, \omega)$. At intermediate doping they reveal for a wide class of cuprates a well defined large Fermi surface (FS) consistent with the Luttinger theorem and similar quasiparticle (QP) dispersion [2]. This seems to imply the validity of the concept of the usual metal with electronic-like FS. Such simple FL picture is in an apparent contradiction with magnetic and transport properties, e.g. electrical conductivity scales with hole concentration, closer to the picture of holes moving in the antiferromagnetic (AFM) background. Moreover, in ARPES the FL interpretation is spoiled by the overdamped character of QP peaks [3,2]. Although a large background makes fits of particular lineshapes non-unique 3,4, the $\mathrm{QP}$ inverse lifetime is found to be of the order of the QP energy, i.e. $\tau^{-1} \propto \omega$ for $\omega>T$, leading to the concept of the marginal Fermi liquid (MFL) [5] with an anomalous single-particle and transport relaxation, in contrast to $\tau^{-1} \propto \omega^{2}$ in the normal FL.

It is unclear whether above features can be reproduced within generic models of strongly correlated systems, such as the Hubbard and the $t-J$ model, in particular in the most challenging regime of intermediate doping. Spectral properties of these 2D models have been so far studied mainly via numerical techniques [6], e.g. exact diagonalization (ED) [7] and Quantum Monte Carlo (QMC) [8]. These studies, as well as some analytical approaches [9], established a reasonable consistency of the model QP dispersion with the experimental one, as well as the possibility of large FS, but have not been able to investigate closer the character of QP, being in the core of the anomalous low-energy properties.

The aim of the present work is to employ the finitetemperature Lanczos method [10] to calculate $A(\mathbf{k}, \omega)$ within the $t-J$ model. This method has been already applied to other dynamic [11] and static [12] functions, yielding features consistent with the MFL concept and experiments on cuprates. Although calculations are still done in small systems, by using finite (but small) $T>0$ smooth enough spectra are obtained not only to determine the QP dispersion, but for the first time also the spectral lineshapes and corresponding self-energies.

We study the $t-J$ model 13

$$
H=-t \sum_{\langle i j\rangle s}\left(\tilde{c}_{j s}^{\dagger} \tilde{c}_{i s}+\text { H.c. }\right)+J \sum_{\langle i j\rangle}\left(\mathbf{S}_{i} \cdot \mathbf{S}_{j}-\frac{1}{4} n_{i} n_{j}\right)
$$

on the planar square lattice and set $J / t=0.3$ to address the regime of cuprates. The operators $\tilde{c}_{j s}, \tilde{c}_{j s}^{\dagger}$ project out the states with doubly occupied sites. The spectral properties of the model Eq. (11) are investigated by calculating the retarded Green's function ( $\mu$ is chemical potential)

$$
G(\mathbf{k}, \omega)=-i \int_{0}^{\infty} d t e^{i(\omega+\mu) t}\left\langle\left\{\tilde{c}_{\mathbf{k} s}(t), \tilde{c}_{\mathbf{k} s}^{\dagger}(0)\right\}\right\rangle .
$$

The average is grandcanonical, which in actual calculations at low $T$ in a system with $N$ sites and fixed hole concentration $c_{h}=N_{h} / N$ is replaced by a canonical one in the subspace of states with $N_{h}$ holes. The two anticommutator terms correspond at low $T$ to the hole inverse photoemission spectra (IPES), and the electron photoemission spectra (PES), respectively.

The calculation of $G(\mathbf{k}, \omega)$ at $T=0$ with the ED technique is well established [7]6], but a small number of sharp peaks in the spectra makes it difficult to extract information on lineshapes and self energies. The QMC methods resort to the use of maximum entropy analysis [8], which also leads to quite restricted $\omega$-resolution. The $T>0$ Lanczos method 10 eliminates these problems for dynamic quantities, i.e. yields smoother spectra and allows for study of the $T$-dependence. The requirement is however that $T>T_{\mathrm{fs}}$, where $T_{\mathrm{fs}}$ is the characteristic temperature at which in a given small system the finitesize effects set in (for discussion of the method we refer to 
previous works [10,11]). We have calculated the Green's function Eq. (2) on systems with $\mathrm{N}=16$ and 18 sites using $\sim 120$ Lanczos steps and sampling over $\sim 1000$ random states. The finite-size effects are small at $T \gtrsim T_{\mathrm{fs}}\left(N, N_{h}\right)$, where, e.g., $T_{\mathrm{fs}} \sim 0.1 t$ for $N_{h} / N=3 / 16$.

From the Green's function we obtain the spectral function $A(\mathbf{k}, \omega)=-(1 / \pi) \operatorname{Im} G(\mathbf{k}, \omega)$ and the one-particle density of states $(\mathrm{DOS}) \mathcal{N}(\varepsilon)=(2 / N) \sum_{\mathbf{k}} A(\mathbf{k}, \varepsilon-\mu)$. The latter is used to define the zero of energy and thus the chemical potential in Eq. (2) via $\int_{-\infty}^{\infty} \mathcal{N}(\omega+\mu)\left(e^{\beta \omega}+\right.$ $1)^{-1} d \omega=1-c_{h}$. We find a very good agreement between $\mu$ calculated this way and from the thermodynamic function $c_{h}(T, \mu)=N_{h} / N$ [12].

Of particular interest is the self-energy

$$
\Sigma(\mathbf{k}, \omega)=\omega-G(\mathbf{k}, \omega)^{-1} .
$$

The relation contains no free term, in contrast to the usual definition, since the $t-J$ model does not allow for a free-fermion propagation even at $J=0$. It is also important to note that due to projected fermion operators in the model the spectral function $A(\mathbf{k}, \omega)$ is not normalized to unity [7], but rather to $\left\langle\left\{\tilde{c}_{\mathbf{k} s}, \tilde{c}_{\mathbf{k} s}^{\dagger}\right\}\right\rangle=\left(1+c_{h}\right) / 2$. This has several consequences, e.g. $\operatorname{Re} \Sigma(\mathbf{k}, \omega \rightarrow \infty)$ does not vanish, but varies linearly with $\omega$.

In Fig. 1 1 we first present $A(\mathbf{k}, \omega)$ for systems with $c_{h} \sim 0.12$ (combining results for $N_{h}=2$ on systems with $N=16,18)$ and $c_{h}=3 / 16$. The spectra are broadened to Lorentzians of variable width $\delta=\delta_{0}+$ $\left(\delta_{\infty}-\delta_{0}\right) \tanh ^{2}(\omega / \Delta)$, with $\delta_{\infty}=0.2 t, \delta_{0}=0.04 t$, and $\Delta=1.0 t$. In this way sharper (well resolved) low-energy features remain unaffected, while the fluctuations at higher $\omega$, mainly due to restricted sampling, are smoothened out. In any case, $\delta$ is always smaller than the energy scale of main spectral features.

We observe in Fig. 1, presented at all available $\mathbf{k}$, a coexistence of sharper features, associated with coherent QP peaks, and of a pronounced incoherent background, as already established in earlier studies [7]. The coherent peaks in Fig. 1 disperse through $\omega=0$ as $\mathbf{k}$ crosses the FS. Within the given resolution in the $\mathbf{k}$-space the FS appears to be large already for $c_{h}=2 / 18$, consistent with the Luttinger theorem. The total QP dispersion $W$ is broadened as $c_{h}$ is increased, qualitatively consistent with the slave boson picture where $W \propto c_{h} t+\chi J$ [9].

In Fig. 2 we show $\Sigma(\mathbf{k}, \omega)$ at $c_{h}=3 / 16$ and at lowest $T=0.1 t \sim T_{\mathrm{fs}}$. We first notice an asymmetry between the PES $(\omega<0)$ and IPES $(\omega>0)$ spectra at all k. $\operatorname{Im} \Sigma$ are small for $\omega>0$, as compared to $\omega<0$. For $\mathbf{k}$ outside FS this implies a weak QP damping, consistent with sharp IPES peaks seen in $A(\mathbf{k}, \omega)$, Fig. 1, containing the major part of the spectral weight. $\operatorname{Re} \Sigma$ shows an analogous asymmetry, in the region $\omega>0$ resembling moderately renormalized QP. Due to projections in Eq. (11), the slope in $\operatorname{Re} \Sigma$ is not zero even at $|\omega| \gg t, J$.

The behavior on the PES $(\omega<0)$ side is very different.
For all $\mathbf{k}, \operatorname{Im} \Sigma$ are very large (several $t$ away from $\omega \sim 0$ ), leading to overdamped QP structures. We should here distinguish two cases. For $\mathbf{k}$ well outside FS, $\operatorname{Im} \Sigma>t$ does not invalidate a well defined QP (at $\omega>0$ ), but rather induces a weaker reflection (shadow) of the peak at $\omega<0$, as well seen in Fig. 1 for $\mathbf{k}=(\pi, \pi)$. On the other hand, the $\omega$ variation for $\mathbf{k}$ inside or near the FS is more regular, and can be directly related to the QP damping. Particularly remarkable feature, found in Fig. 2, is a linear frequency dependence of $\operatorname{Im} \Sigma$ at $\omega<0$ for $\mathbf{k}=(\pi / 2,0),(\pi / 2, \pi / 2)$. Meanwhile $\mathbf{k}=(0,0)$, being further away from the FS, seems to follow a different (more FL-type) behavior. Such general behavior remains similar also for the lower doping $c_{h}=2 / 18$.

To address the latter point in more detail, we show in Fig. 3 the $T$-variation of $\operatorname{Im} \Sigma$ for both dopings at selected $\mathbf{k}$ below the FS. For $c_{h}=3 / 16$ the linearity of $\operatorname{Im} \Sigma(\omega)$ is seen in a broad range $-2 t \lesssim \omega \lesssim 0$ at the lowest $T$ shown. Moreover, for this higher ('optimum') doping the $T$-dependence is close to a linear one, assuming a small residual (finite-size) damping $\eta_{0}$ at $\omega=0$. Data can be well described by $\operatorname{Im} \Sigma=\eta_{0}+\gamma(|\omega|+\xi T)$, with $\gamma \sim 1.4$ and $\xi \sim 3.5$, baring a similarity to the MFL ansatz [5], as well as to the conductivity relaxation $\tau_{c}^{-1}$ found in the $t-J$ model 11]. In contrast, the $T$-dependence for $c_{h}=$ $2 / 18$ seems somewhat different, and $\operatorname{Im} \Sigma \propto \omega$ only in the interval $-t \lesssim \omega \lesssim T$. This would indicate the consistency with the alternative MFL form [5], however we should be aware that in this 'underdoped' regime finite-size effects are larger at fixed $T$.

Here we should comment on the manifestation of the FS in small correlated systems. At $T, \omega \sim 0$ we are dealing in the evaluation of Eq.(2) with the transition between ground states of systems with $N_{h}$ and $N_{h}^{\prime}=N_{h} \pm 1$ holes, respectively. Since these states have definite momenta $\mathbf{k}_{0}$, they induce strong QP peaks for particular $\mathbf{k}=\mathbf{k}_{0}^{\prime}-\mathbf{k}_{0}$ (defining in this way for a small system the FS, apparently satisfying the Luttinger theorem), with $\operatorname{Im} \Sigma(\mathbf{k}, \omega \sim 0) \sim 0$. However, the calculated $T$-variation is for a given system meaningful only at $T>T_{\mathrm{fs}}$.

From $\Sigma(\mathbf{k}, \omega)$ we can calculate QP parameters: the dispersion $E_{\mathbf{k}}$, the weight $Z_{\mathbf{k}}$ and the damping $\Gamma_{\mathbf{k}}$,

$$
\begin{aligned}
E_{\mathbf{k}} & =\operatorname{Re} \Sigma\left(\mathbf{k}, E_{\mathbf{k}}\right), \\
Z_{\mathbf{k}} & =[1-\partial \operatorname{Re} \Sigma(\mathbf{k}, \omega) / \partial \omega]_{\omega=E_{\mathbf{k}}}^{-1}, \\
\Gamma_{\mathbf{k}} & =Z_{\mathbf{k}}\left|\operatorname{Im} \Sigma\left(\mathbf{k}, E_{\mathbf{k}}\right)\right|,
\end{aligned}
$$

which are listed in Table 1 . We note that parameters are of a limited meaning for $\mathbf{k}$ inside FS due to large $\Gamma$. In particular, $E_{\mathbf{k}}$ (as well as $Z_{\mathbf{k}}$ and $\Gamma_{\mathbf{k}}$ ) for $\mathbf{k}=(0,0)$ do not correspond to a weak QP peak at $\omega \sim-t$, being overwhelmed by the incoherent background. Otherwise, the enhancement of the dispersion with $c_{h}$ is seen, accompanied by a decrease of $\Gamma$ for $|\mathbf{k}|>k_{F}$. To establish the relation with the FL theory one has to evaluate QP parameters at the FS, $\mathbf{k}=\mathbf{k}_{F}$. Of particular importance is the 
renormalization factor $\tilde{Z}=Z_{\mathbf{k}_{F}}$. $\tilde{Z}$ is still decreasing as $T$ is lowered. Nevertheless we find a weak variation (cca. $20 \%$ ) within the interval $0.1<T / t<0.3$, not inconsistent with the MFL form, leading to $\tilde{Z}^{-1} \sim \ln \left(\omega_{c} / T\right)$. Regarding the size of $\tilde{Z}$ (at low but finite $T>0$ ) we note, that the value of the momentum distribution function $\bar{n}_{\mathbf{k} s}$ is very close to the maximum for the $t-J$ model, $\bar{n}_{\mathbf{k s}} \sim\left(1+c_{h}\right) / 2$, for all $|\mathbf{k}|<k_{F}$ [7]. Taking the FS volume according to Luttinger theorem and assuming that $\bar{n}_{\mathbf{k} s}$ falls monotonously with $|\mathbf{k}|$, this implies the discontinuity $\tilde{Z}=\delta \bar{n}_{\mathbf{k} s}<2 c_{h} /\left(1+c_{h}\right)$. We indeed find a consistent result $\tilde{Z}=0.28$ for $c_{h}=3 / 16$, while for $c_{h}=2 / 18$ the value is still larger, possibly due to too high $T$.

An analogous argument can be used to explain the electron-hole asymmetry of $A(\mathbf{k}, \omega)$. Holes added to the system at $|\mathbf{k}|<k_{F}, \omega<0$ move in an extremely correlated system, strongly coupled to the spin dynamics [14], also following the anomalous low- $\omega$ behavior [11. On the other hand, states for $|\mathbf{k}|>k_{F}$ are not fully populated, allowing for a moderately damped motion of added electrons for $\omega>0$.

Another feature is seen predominantly at smaller doping $c_{h} \sim 0.12$ for $|\mathbf{k}|>k_{F}$ : along with the principal peak at $\omega>0$ a weak bump in the $\omega<0$ part of the spectrum appears when the FS is crossed along $\overline{\Gamma M}$. In $\operatorname{Re} \Sigma$ for $\mathbf{k}=(\pi, \pi)$ it emerges even as a strong oscillation, leading to a double solution in Eq. (何 [15]. In ARPES this should be seen as the reappearance of the 'shadow' QP band for $\mathbf{k}$ above the FS, in accordance with experiments [1] and some previous studies [15,7]. The effect is less pronounced at larger doping $c_{h}=3 / 16$, probably due to the reduction of the AFM correlation length.

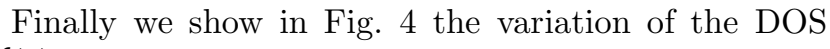
$\mathcal{N}(\varepsilon)$ with doping. For a hole injected in the weakly doped system $\left(c_{h} \sim 0.06\right)$, a QP coherent peak (of width $\sim 2 J$ ) is seen at $\varepsilon \lesssim \mu$. Besides, a broad background (due to well understood incoherent hole motion) is dominating lower $\varepsilon$. At such low doping the electron part of DOS is weaker, with the total intensity $2 c_{h}$ as compared to $1-c_{h}$ of the hole part. With increasing $c_{h}$ the hole background doesn't reduce in intensity, while the coherent peak near the Fermi energy widens and its spectral weight reduces, reflecting the broadening of the QP dispersion. At the same time, the electron part of DOS is increasing, both in the weight and in the width. Note that oscillations for $\varepsilon>\mu$ appear in this regime due to the underdamped QP and a restricted number of finite-size $\mathbf{k}$.

Here we mention the relation with the entropy $s$ [12], assuming the low- $T$ form as follows from the FL theory [16], i.e. $s=\pi^{2} T \mathcal{N}(\mu) / 3 \tilde{Z}$. With $\mathcal{N}(\mu)$ in Fig. 田, weakly doping dependent at intermediate $c_{h}$ (also quite close to the free-fermion value), and $\tilde{Z} \sim 0.28$ for $c_{h}=3 / 16$, we get $s \sim 0.29 k_{B} /$ site at $T=0.1 t$, consistent with static calculations [12]. Nevertheless, one should keep in mind that such $s$ represents a large increase over the undoped (AFM) system, taking into account very few mobile holes introduced into the system.

In the end we comment on the relevance of our results to the understanding of the ARPES spectra in cuprates [1]. For $\omega<0$ we notice the importance of the incoherent background, consistent with the observation that in fitting the experiments to either FL or MFL form an


$|\mathbf{k}|<k_{F}$ we find the linewidth typically $\Gamma \sim t$ (see Table [i), well compatible ( $t \sim 0.4 \mathrm{eV}$ in cuprates [17, 13] $)$ with experiments at $\mathbf{k}$ away from the FS and at intermediate doping [1,2]. Also the MFL form has been claimed to describe better the experiments [3], although this point is not yet clarified 1 . We note also that our QP dispersion and the shape of the FS are not entirely of the form found experimentally [1]. This could be possibly remedied by including the n.n.n. $\left(t^{\prime}\right)$ hopping term 17]. Still we do not expect such corrections to modify conclusions concerning the spectral shapes and the QP character.

One of the authors (P.P.) wishes to thank P. Horsch, G. Khalliulin, R. Zeyher, and T.M. Rice for useful suggestions and fruitful discussions, and acknowledges the support of the MPI für Festkörperphysik, Stuttgart, where a part of this work has been performed.

[1] For reviews, see Z.-X. Shen and D.S. Dessau, Phys. Rep. 253, 1 (1995); Z.-X. Shen et al., Science 267, 343 (1995).

[2] H. Ding et al., Phys. Rev. Lett. 76, 1533 (1996); D.S. Marshall et al., ibid., 76, 4841 (1996).

[3] C.G. Olson et al., Phys. Rev. B 42, 381 (1990).

[4] L.Z. Liu, R.O. Anderson, and J.W. Allen, J. Phys. Chem. Solids 52, 1473 (1991).

[5] C.M. Varma et al., Phys. Rev. Lett. 63, 1996 (1989); P.B. Littlewood and C. M. Varma, J. Appl. Phys. 69, 4979 (1991).

[6] For a review, see E. Dagotto, Rev. Mod. Phys. 66, 763 (1994).

[7] W. Stephan and P. Horsch, Phys. Rev. Lett. 66, 2258 (1991); A. Moreo, S. Haas, A.W. Sandvik, and E. Dagotto, Phys. Rev. B 51, 12045 (1995).

[8] N. Bulut, D.J. Scalapino, and S.R. White, Phys. Rev. B 50, 7215 (1994); R. Preuss, W. Hanke, and W. von der Linden, Phys. Rev. Lett. 75, 1344 (1995).

[9] G. Baskaran, Z. Zou, and P.W. Anderson, Solid State Commun. 63, 973 (1987); Z. Wang, Y. Bang, and G. Kotliar, Phys. Rev. Lett. 67, 2733 (1991).

[10] J. Jaklič and P. Prelovšek, Phys. Rev. B 49, 5065 (1994).

[11] J. Jaklič and P. Prelovšek, Phys. Rev. Lett. 74, 3411 (1995); ibid., 75, 1340 (1995); Phys. Rev. B 52, 6903 (1995).

[12] J. Jaklič and P. Prelovšek, Phys. Rev. Lett. (to be published), cond-mat/9603081.

[13] For a review, see T.M. Rice, in High Temperature Superconductivity, Proceedings of the 39th Scottish Universi- 
ties Summer School in Physics, edited by D.P. Turnball and W. Barford (Adam Hilger, London, 1991), p.317.

[14] P. Prelovšek, to be published.

[15] A.P. Kampf and J.R. Schrieffer, Phys. Rev. B 41, 6399 (1990); ibid. 42, 7967 (1990).

[16] A.A. Abrikosov, L.P. Gor'kov, and I.E. Dzyaloshinskii, Quantum Field Theoretical Methods in Statistical Physics (Pergamon, Oxford, 1965), p. 169.

[17] M.S. Hybertsen et al., Phys. Rev. B 41, 11068 (1990); T. Tohyama and S. Maekawa, Phys. Rev. B 49, 3596 (1994); A. Nazarenko et al, Phys. Rev. B 51, 8676 (1995).

FIG. 1. Spectral functions for $N_{h}=2$ holes on systems with $N=16,18$ sites, and for $N_{h}=3$ holes on $N=16$ sites.

FIG. 2. Self energy for $c_{h}=3 / 16$ at various $\mathbf{k}$.

FIG. 3. $\operatorname{Im} \Sigma(\mathbf{k}, \omega)$ for various $T$ for the systems with $c_{h}=2 / 18$ and $c_{h}=3 / 16$ at selected $\mathbf{k}$ below the FS.

FIG. 4. Single-particle DOS $\mathcal{N}(\varepsilon)$ for variously doped systems. For $c_{h} \sim 0.06$ and $c_{h} \sim 0.12$ we present joined densities for $N=16,18$ systems with $N_{h}=1$ and $N_{h}=2$, respectively. The thin vertical lines denote the Fermi energy $\mu$.

TABLE I. QP parameters for two hole concentrations.

\begin{tabular}{cccccccc}
\hline \hline \multicolumn{3}{c}{$c_{h}=2 / 18, T / t=0.15$} & \multicolumn{3}{c}{$c_{h}=3 / 16, T / t=0.15$} \\
$\mathbf{k}$ & $E_{\mathbf{k}} / t$ & $Z_{\mathbf{k}}$ & $\Gamma_{\mathbf{k}} / t$ & $\mathbf{k}$ & $E_{\mathbf{k}} / t$ & $Z_{\mathbf{k}}$ & $\Gamma_{\mathbf{k}} / t$ \\
\hline$(0,0)$ & -3.8 & 0.80 & 1.9 & $(0,0)$ & -4.2 & 0.73 & 1.4 \\
$(\pi / 3, \pi / 3)$ & -0.7 & 0.26 & 0.65 & $(\pi / 2,0)$ & -1.1 & 0.68 & 1.7 \\
$(2 \pi / 3,0)$ & -0.4 & 0.35 & 0.51 & $(\pi / 2, \pi / 2)$ & 0.0 & 0.28 & 0.32 \\
$(2 \pi / 3,2 \pi / 3)$ & 0.5 & 0.35 & 0.49 & $(\pi, 0)$ & 0.0 & 0.28 & 0.32 \\
$(\pi, \pi / 3)$ & 0.1 & 0.26 & 0.40 & $(\pi, \pi / 2)$ & 0.8 & 0.44 & 0.31 \\
$(\pi, \pi)$ & 1.1 & 0.37 & 0.54 & $(\pi, \pi)$ & 1.7 & 0.46 & 0.35 \\
\hline \hline
\end{tabular}




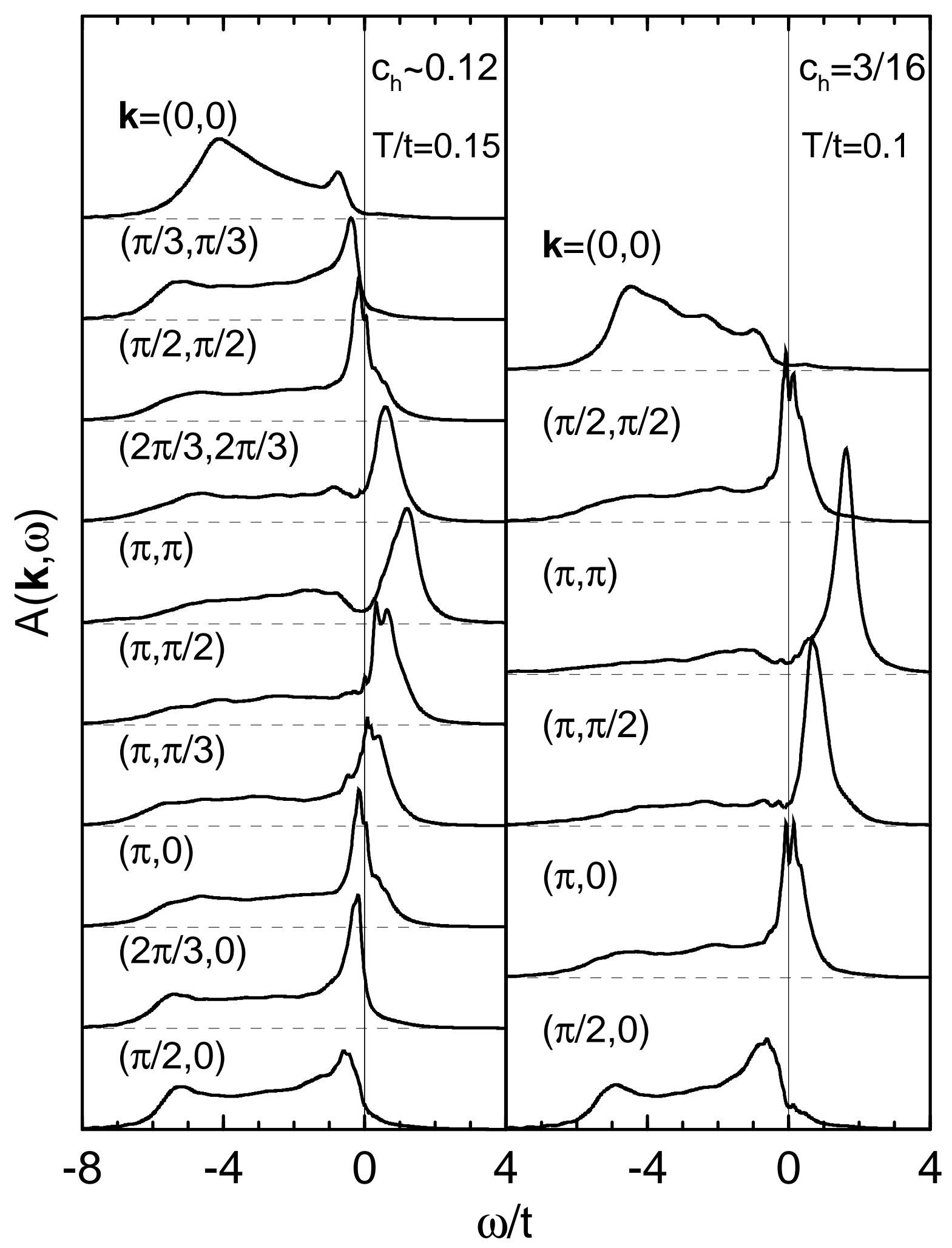




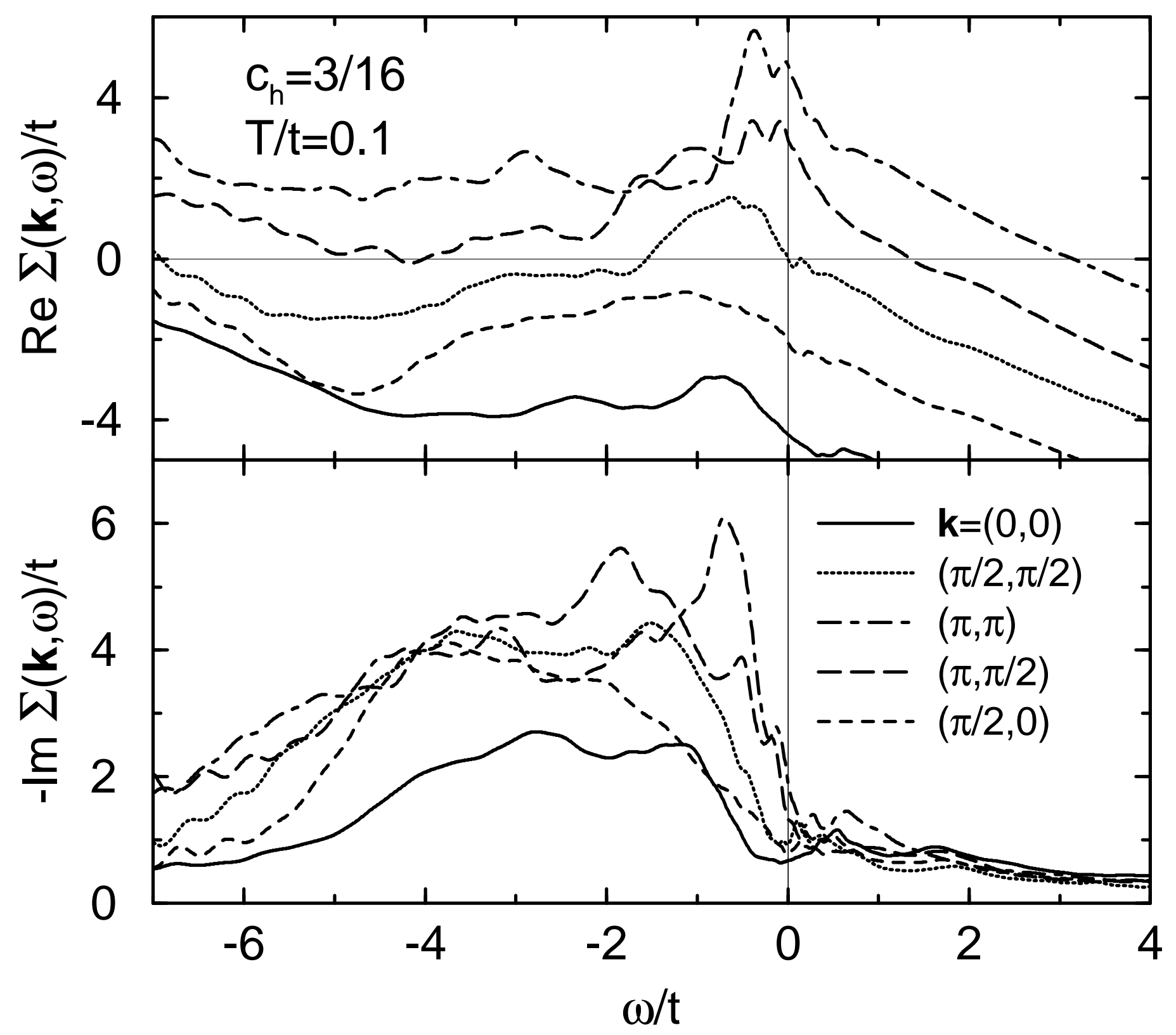




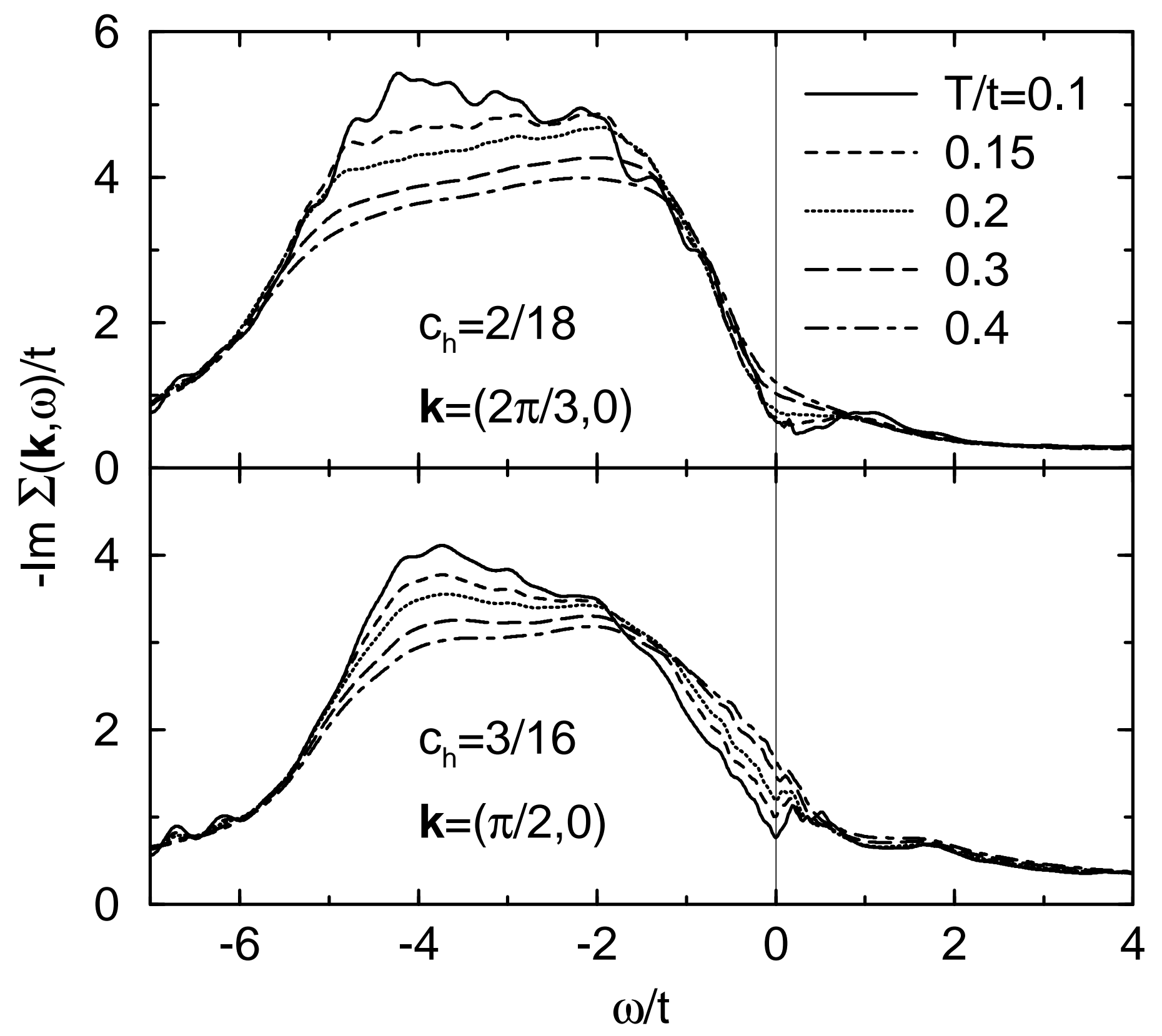




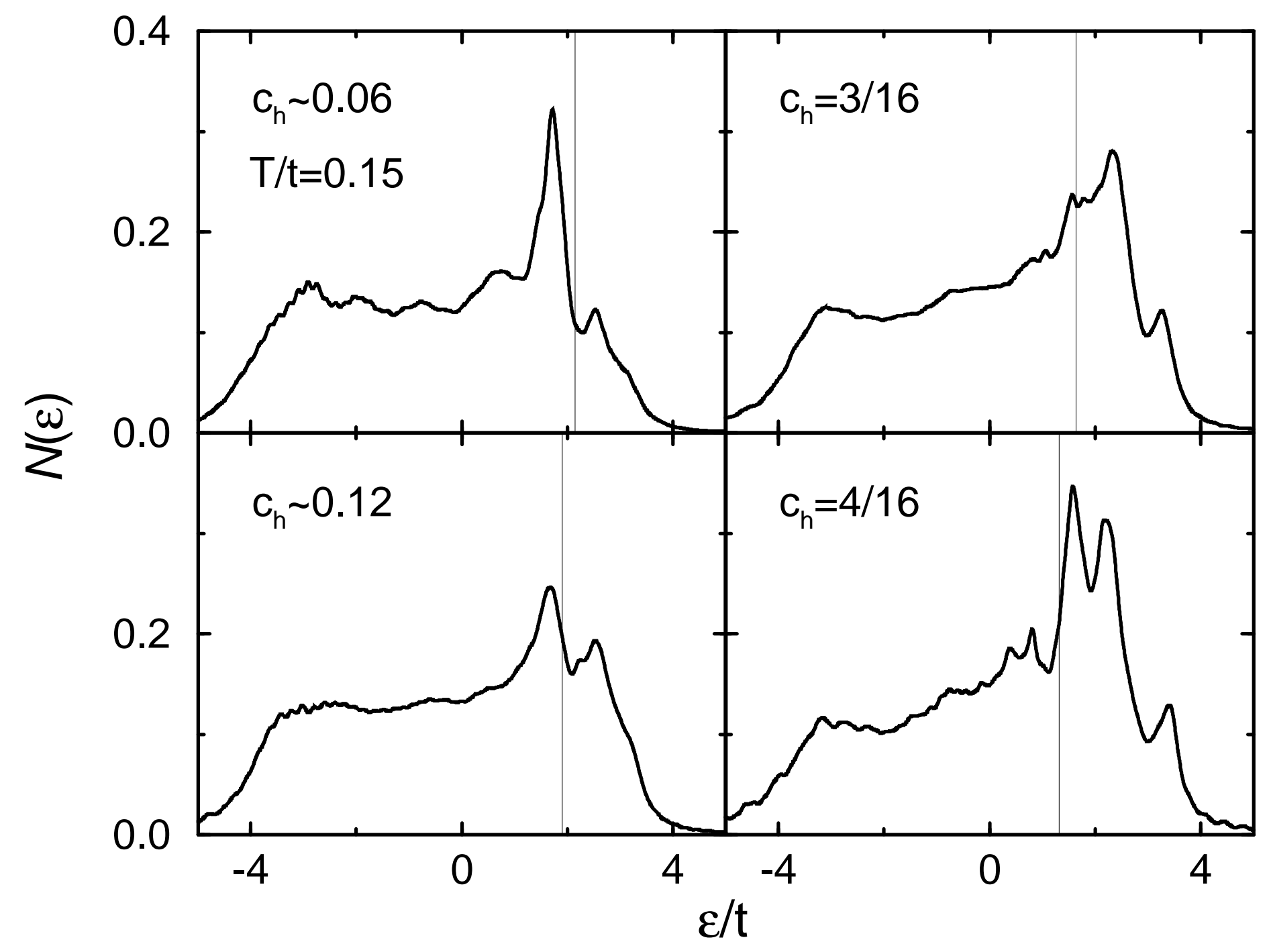

